\title{
Lagrangian relaxation bounds for a production-inventory-routing problem *
}

\author{
Agostinho Agra $^{1}$, Adelaide Cerveira ${ }^{2}$, and Cristina Requejo ${ }^{1}$ \\ 1 University of Aveiro and CIDMA, 3810-193 Aveiro, Portugal, \\ \{aagra, crequejo\}@ua.pt \\ 2 University of Trás-os-Montes e Alto-Douro, 5001-801 Vila Real and INESC TEC, Portugal \\ cerveira@utad.pt
}

\begin{abstract}
We consider a single item Production-Inventory-Routing problem with a single producer/supplier and multiple retailers. Inventory management constraints are considered both at the producer and at the retailers, following a vendor managed inventory approach, where the supplier monitors the inventory at retailers and decides on the replenishment policy for each retailer. We assume a constant production capacity. Based on the mathematical formulation we discuss a classical Lagrangian relaxation which allows to decompose the problem into four subproblems, and a new Lagrangian decomposition which decomposes the problem into just a production-inventory subproblem and a routing subproblem. The new decomposition is enhanced with valid inequalities. A computational study is reported to compare the bounds from the two approaches.
\end{abstract}

Keywords: Inventory routing; Lagrangian relaxation; Lagrangian decomposition; Lower bounds.

\section{Introduction}

We consider a single item Production-Inventory-Routing (PIR) problem with a single producer/supplier and multiple clients/retailers. Such complex problems combining production, inventory and routing decisions have been receiving a great attention in recent years with a large increase of the number of publications. Applications to complex supply chain problems can be found, for instance, in $[3,4,6,10,13,16,19]$. For surveys on complex inventory routing problems covering theoretical and industrial aspects see $[1,7,11]$. The problem considered in this paper fits in the land transportation mode since for each time period a vehicle routing problem is solved in order to find the low cost distribution plan for the deliveries assigned to that period. This contrasts with maritime transportation where, in general, a route may take several time periods $[3,4,6,10]$.

In the past, such complex problems were typically decomposed and solved separately. Lagrangian relaxations for related problems have been used in $[9,15,23]$ where the problem is decomposed into several simpler subproblems. Recent works have shown that there are gains in considering the interrelated problems simultaneously in order to coordinate the different decisions $[5,20,23]$. There are a few papers reporting exact algorithms to solve these problems. Such exact algorithms are based on branch-and-cut procedures. Some examples can be found

\footnotetext{
* Published in Machine Learning, Optimization, and Big Data: Second International Workshop, MOD 2016, Revised Selected Papers. Lecture Notes in Computer Science, Volume 10122, 236-245, 2016.
} 
in $[3,4,8,20,22]$. In order to solve efficiently such problems by branch-and-cut it is important to use good formulations [3,4]. Therefore, some relevant work has been done on the modeling of complex problems, either by including valid inequalities or by using extended formulations, see $[2,3,4,5,13,20,22,24]$.

We consider a classical model for the PIR problem and discuss a Lagrangian relaxation and a Lagrangian decomposition. First we adapt the Lagrangian relaxation given by Fumero and Vercellis in [15], that decomposes the problem into (i) a trivial production subproblem at the supplier, (ii) a trivial lotsizing problem at the supplier, (iii) a lotsizing problem at each client, and (iv) a capacitated vehicle routing problem in each time period. Then we propose a new Lagrangian decomposition that uses a duplication of variables. The problem is decomposed into (i) a subproblem that considers the production at the supplier and the inventory management at the supplier and clients, and (ii) several vehicle routing subproblems, one for each time period. The Lagrangian decomposition is enhanced with the inclusion of valid inequalities. Although the subproblems are more complex than the subproblems resulting from the classical Lagrangian relaxation, computational tests show that by considering such more complex subproblems (all of them are NP-hard) one obtains better bounds when the subproblems can be solved to optimality.

The outline of the paper is as follows. In Section 2 we introduce a mixed integer formulation to the PIR problem. In Section 3 we present a Lagrangian relaxation that is similar to the one given in [15]. Then, in Section 4 we introduce a new Lagrangian decomposition and discuss enhancements to tighten each subproblem resulting from the decomposition. In Section 5 the details of benchmark instances generation are given, and computational results to test the model and compare the Lagrangian relaxation with the Lagrangian decomposition are presented. Finally, the conclusions are stated in Section 6.

\section{Mathematical model}

In this section we introduce a mixed integer formulation to the PIR problem.

Consider parameters $n, n t$, and $m$ representing the number of clients, the number of periods, and the number of available vehicles, respectively. Define the sets $N_{c}=\{1, \ldots, n\}$, and $T=\{1, \ldots, n t\}$ representing the set of clients and the set of periods, respectively. Considering node 0 as the producer, $N=\{0\} \cup N_{c}$ is the set of nodes (producer and clients), and $A=\{(i, j): i, j \in N, i \neq j\}$ is the set of arcs. Additionally, consider the following parameters: $d_{i t}$ is the demand of client $i \in N_{c}$ in period $t \in T ; S_{i}^{0}$ is the initial stock at node $i \in N ; \bar{I}_{i}$ is the inventory limit at node $i \in N ; \bar{P}_{t}$ is the production capacity in period $t \in T ; Q$ is the capacity vehicles; $\bar{Q}_{i t}=\min \left\{Q, \sum_{\ell \in t \ldots n t} d_{i \ell}, d_{i t}+\bar{I}_{i}\right\}$ is the delivery quantity limit in period $t \in T$ at client $i \in N_{c}$. For cost parameters, $S C$ is the set up cost for producing in a period, $P C$ is the unit production cost, $V C$ is the cost for using a vehicle, $H_{i}$ is the unit holding cost at node $i \in N$, and $C_{i j}$ is the traveling cost from node $i$ to node $j,(i, j) \in A$.

For the production and inventory decision we define the following variables: $y_{t}$ is a binary variable that indicates whether there is production in period $t \in T$ or not; $p_{t}$ is the production level in period $t \in T$; $s_{i t}$ is the stock level at node $i \in N$ at the end of period $t \in T ; q_{i t}$ indicates the quantity delivered at node $i \in N_{c}$ in period $t \in T$. For the routing decisions we define the variables: $x_{i j t}$ indicates whether a vehicle travels in $\operatorname{arc}(i, j) \in A$ in period $t \in T$ or not; $z_{i t}$ is a binary variable that is one if node $i \in N_{c}$ is visited in period $t \in T$; $v_{t}$ indicates the number of vehicles leaving the producer in period $t \in T$, and $f_{i j t}$ is the quantity of product transported in $\operatorname{arc}(i, j) \in A$ in period $t \in T$. 
The PIR model is as follows:

$$
\begin{aligned}
& \min \sum_{t \in T} S C y_{t}+\sum_{t \in T} P C p_{t}+\sum_{t \in T} \sum_{i \in N} H_{i} s_{i t}+\sum_{t \in T} \sum_{(i, j) \in A} C_{i j} x_{i j t}+\sum_{t \in T} V C v_{t} \\
& \text { subject to } S_{0}^{0}+p_{t}=\sum_{i \in N_{c}} q_{i t}+s_{0 t} \quad t=1 \\
& s_{0, t-1}+p_{t}=\sum_{i \in N_{c}} q_{i t}+s_{0 t} \quad \forall t \in T, t>1 \\
& S_{i}^{0}+q_{i t}=d_{i t}+s_{i t} \quad \forall i \in N_{c}, t=1 \\
& s_{i, t-1}+q_{i t}=d_{i t}+s_{i t} \quad \forall i \in N_{c}, \forall t \in T, t>1 \\
& s_{i t} \leq \bar{I}_{i} \\
& \forall i \in N, \forall t \in T \\
& p_{t} \leq \bar{P}_{t} y_{t} \\
& \forall t \in T \\
& q_{i t} \leq \bar{Q}_{i t} z_{i t} \\
& \forall i \in N_{c}, \forall t \in T \\
& \sum_{j \in N} x_{i j t}=z_{i t} \quad \forall i \in N_{c}, \forall t \in T \\
& \sum_{j \in N} x_{j i t}+\sum_{j \in N} x_{i j t}=2 z_{i t} \quad \forall i \in N, \forall t \in T \\
& \sum_{j \in N} x_{0 j t}=v_{t} \quad \forall t \in T \\
& v_{t} \leq m \quad \forall t \in T \\
& \sum_{i \in N} f_{i j t}-\sum_{i \in N_{c}} f_{j i t}=q_{j t} \quad \forall j \in N_{c}, \forall t \in T \\
& f_{i j t} \leq Q x_{i j t} \\
& \forall(i, j) \in A, \forall t \in T \\
& y_{t}, z_{i t}, x_{i j t} \in\{0,1\} \\
& \forall i, j \in N, \forall t \in T \\
& v_{t}, f_{i j t} \in \mathbb{Z}^{+} \\
& \forall i, j \in N, \forall t \in T \\
& s_{i t}, p_{t}, q_{i t} \geq 0 \\
& \forall i \in N, \forall t \in T
\end{aligned}
$$

The objective function (1) is to minimize the total cost which includes the production set-up, the production, the holding, the traveling, and the vehicle usage costs. Constraints (2) and (3) are the inventory conservation constraints at the producer, and (4) and (5) are the inventory conservation constraints at clients. Constraints (6) impose a storage capacity at the producer and at each client. Constraints (7) impose limits on the production at each period, and constraints (8) impose limits on the delivery quantity at each client for each period. Constraints (9) and (10) are the routing constraints. Constraints (11) together with constraints (12) guarantee that the number of vehicles leaving the producer does not exceeds the available number of vehicles. Constraints (13) are the flow balance constraints at clients and constraints (14) guarantee that the capacity of each vehicle is not exceeded. Constraints (15), (16), and (17) are the variables domain constraints.

\section{Lagrangian relaxation}

Here we present a Lagrangian relaxation that follows the one from Fumero and Vercelis [15], however in [15] constraints (8) are not considered. In order to derive the Lagrangian relaxation 
we associate multipliers $\lambda_{t}^{S}$ to constraints (2) (for $t=1$ ) and (3) (for $t>1$ ), nonnegative multipliers $\lambda_{i t}^{D}$ to constraints (8) and nonnegative multipliers $\lambda_{i j t}^{F}$ to constraints (14) and dualize these constraints in the usual Lagrangian way.

This leads to the following relaxation

$$
\begin{aligned}
\mathscr{L}\left(\lambda^{S}, \lambda^{D}, \lambda^{F}\right)= & \min \sum_{t \in T} S C y_{t}+\sum_{t \in T} P C p_{t}+\sum_{t \in T} \sum_{i \in N} H_{i} s_{i t}+\sum_{t \in T} \sum_{(i, j) \in A} C_{i j} x_{i j t}+\sum_{t \in T} V C v_{t}+ \\
& +\lambda_{1}^{S}\left(S_{0}^{0}+p_{1}-\sum_{i \in N} q_{i 1}-s_{01}\right)+\sum_{t \in T, t>1} \lambda_{t}^{S}\left(s_{0, t-1}+p_{t}-\sum_{i \in N} q_{i t}-s_{0 t}\right)+ \\
& +\sum_{t \in T} \sum_{i \in N_{c}} \lambda_{i j t}^{D}\left(q_{i t}-\bar{Q}_{i t}\right)+\sum_{t \in T} \sum_{(i, j) \in A} \lambda_{i j t}^{F}\left(f_{i j t}-Q x_{i j t}\right)
\end{aligned}
$$

subject to $(4)-(13),(15)-(17)$.

This problem can be separated into four subproblems: a production subproblem (on variables $y_{t}, p_{t}$ ), an inventory subproblem at the supplier (on variables $s_{0 t}$ ), an inventory subproblem at the retailers (on variables $s_{i t}, q_{i t}, f_{i j t}$ ) and a routing subproblem (on variables $x_{i j t}, z_{i t}, v_{t}$ ). The first two subproblems can de solved by inspection.

For each set of multipliers $\left(\lambda^{S}, \lambda^{D}, \lambda^{F}\right)$, with $\lambda^{S} \in \mathbb{R}, \lambda^{D} \in \mathbb{R}_{0}^{+}$, and $\lambda^{F} \in \mathbb{R}_{0}^{+}$, the value of $\mathscr{L}\left(\lambda^{S}, \lambda^{D}, \lambda^{F}\right)$ gives a lower bound on the optimum value of the PIR problem. To obtain the best lower bound the following dual Lagrangian problem: $\max _{\lambda^{S} \in \mathbb{R}, \lambda^{D} \geq 0, \lambda^{F} \geq 0} \mathscr{L}\left(\lambda^{S}, \lambda^{D}, \lambda^{F}\right)$, has to be solved.

A subgradient optimization procedure [17] is used to solve the dual Lagrangian problem. This procedure starts by initializing the Lagrangian multipliers $\left(\lambda^{S}, \lambda^{D}, \lambda^{F}\right)$ to $\left(\lambda_{0}^{S}, \lambda_{0}^{D}, \lambda_{0}^{F}\right)$. We set $\left(\lambda_{0}^{S}\right)_{t}=S_{0}^{0}, t \in T,\left(\lambda_{0}^{S}\right)_{n t+1}=0,\left(\lambda_{0}^{D}\right)_{i t}=\left(S_{0}^{0}+n\right) / \bar{Q}_{i t}, i \in N_{c}, t \in T$ and $\left(\lambda_{0}^{F}\right)_{i j t}=1 / Q,(i, j) \in A, t \in T$. Then, iteratively, at each iteration $k$, the relaxed problem $\mathscr{L}\left(\lambda_{k}^{S}, \lambda_{k}^{D}, \lambda_{k}^{F}\right)$ is solved and the Lagrangian multipliers are updated using a direction $\left(d_{k}^{S}, d_{k}^{D}, d_{k}^{F}\right)$ and a step-size $\left(s_{k}^{S}, s_{k}^{D}, s_{k}^{F}\right)$ as follows:

$$
\lambda_{k+1}^{S}=\max \left\{\lambda_{k}^{S}+s_{k}^{S} d_{k}^{S}\right\}, \lambda_{k+1}^{D}=\max \left\{\lambda_{k}^{D}+s_{k}^{D} d_{k}^{D}\right\}, \text { and } \lambda_{k+1}^{F}=\max \left\{\lambda_{k}^{F}+s_{k}^{F} d_{k}^{F}\right\} .
$$

The direction, following Held, Wolfe and Crowder [17], is updated as follows:

$$
d_{k}^{S}=\nabla_{k}^{S}, d_{k}^{D}=\nabla_{k}^{D}, \text { and } d_{k}^{F}=\nabla_{k}^{F},
$$

considering the following subgradients: $\left(\nabla_{k}^{S}\right)_{t}=S_{0}^{0}+p_{t}-\sum_{i \in N_{c}} q_{i t}-s_{0 t}, \quad t=1, \quad\left(\nabla_{k}^{S}\right)_{t}=$ $s_{0, t-1}+p_{t}-\sum_{i \in N_{c}} q_{i t}-s_{0 t}, t \in T, t>1, \quad\left(\nabla_{k}^{D}\right)_{i t}=q_{i t}-\bar{Q}_{i t}, i \in N_{c}, t \in T$ and $\left(\nabla_{k}^{F}\right)_{i j t}=$ $f_{i j t}-Q x_{i j t},(i, j) \in A, t \in T$. For the step-size, following Shor [21], we consider:

$$
s_{k}^{S}=\rho \frac{U B-\mathscr{L}\left(\lambda_{k}^{S}, \lambda_{k}^{D}, \lambda_{k}^{F}\right)}{\nabla_{k}^{S} d_{k}^{S}}, s_{k}^{D}=\rho \frac{U B-\mathscr{L}\left(\lambda_{k}^{S}, \lambda_{k}^{D}, \lambda_{k}^{F}\right)}{\nabla_{k}^{D} d_{k}^{D}}, s_{k}^{F}=\rho \frac{U B-\mathscr{L}\left(\lambda_{k}^{S}, \lambda_{k}^{D}, \lambda_{k}^{F}\right)}{\nabla_{k}^{F} d_{k}^{F}}
$$

with $\rho \in] 0,2[$, and where $U B$ is the best upper bound known.

\section{Lagrangian decomposition}

In this section a Lagrangian decomposition of the PIR model is considered, where variables $q_{i t}$ and $z_{i t}$ are duplicated. New variables $q_{i t}^{1}$ replace $q_{i t}$ in constraints (2)-(5), variables $z_{i t}^{2}$ 
replace $z_{i t}$ in constraints $(9)-(10)$, and variables $q_{i t}^{2}$ replace $q_{i t}$ in constraints (13). Constraints (8) are also duplicated and replaced by the following two sets of constraints

$$
\begin{aligned}
& q_{i t}^{1} \leq \bar{Q}_{i t} z_{i t}^{1}, \quad \forall i \in N_{c}, \forall t \in T, \\
& q_{i t}^{2} \leq \bar{Q}_{i t} z_{i t}^{2}, \quad \forall i \in N_{c}, \forall t \in T .
\end{aligned}
$$

Additionally the following equalities are added

$$
\begin{aligned}
& q_{i t}^{1}=q_{i t}^{2} \quad \forall i \in N_{c}, \forall t \in T \\
& z_{i t}^{1}=z_{i t}^{2} \quad \forall i \in N_{c}, \forall t \in T
\end{aligned}
$$

Associating multipliers $\lambda^{q}$ to constraints (20) and multipliers $\lambda^{z}$ to constraints (21), and dualizing these constraints on the Lagrangian way, two separated subproblems are obtained: a production-inventory subproblem, and a routing subproblem.

The production-inventory subproblem corresponds to the relaxation

$$
\mathscr{L}^{P I}\left(\lambda^{q}, \lambda^{z}\right)=\min \sum_{t \in T} S C y_{t}+\sum_{t \in T} P C p_{t}+\sum_{t \in T} \sum_{i \in N} H_{i} s_{i t}+\sum_{t \in T} \sum_{i \in N_{c}}\left(\lambda_{i t}^{q} q_{i t}^{1}+\lambda_{i t}^{z} z_{i t}^{1}\right)
$$

subject to the modified constraints $(2)-(7)$ and (18), with variables $z_{i t}$ and $q_{i t}$ replaced by $z_{i t}^{1}$ and $q_{i t}^{1}$, respectively. While the routing subproblem corresponds to the relaxation

$$
\mathscr{L}^{R}\left(\lambda^{q}, \lambda^{z}\right)=\min \sum_{t \in T} \sum_{(i, j) \in A} C_{i j} x_{i j t}+\sum_{t \in T} V C v_{t}-\sum_{t \in T} \sum_{i \in N_{c}}\left(\lambda_{i t}^{q} q_{i t}^{2}+\lambda_{i t}^{z} z_{i t}^{2}\right)
$$

subject to the modified constraints (9)-(14) and (19), with variables $z_{i t}$ and $q_{i t}$ replaced by $z_{i t}^{2}$ and $q_{i t}^{2}$, respectively. This routing subproblem can be further separated into $n t$ routing subproblems, one for each period.

All these subproblems are NP-hard but can be solved in general for reasonable size instances. For each set of multipliers $\left(\lambda^{q}, \lambda^{z}\right)$ the value of $\mathscr{L}^{P I}\left(\lambda^{q}, \lambda^{z}\right)+\mathscr{L}^{R}\left(\lambda^{q}, \lambda^{z}\right)$ gives a lower bound on the optimum value of the PIR problem. To obtain the best lower bound the corresponding dual Lagrangian problem has to be solved. A subgradient optimization procedure [17] as described above is used. The multipliers are initialized as follows: $\left(\lambda_{0}^{z}\right)_{i t}=C_{0 i}, i \in$ $N_{c}, t \in T,\left(\lambda_{0}^{q}\right)_{i t}=\left(S_{i}^{0}+n\right) / \bar{Q}_{i t}, i \in N_{c}, t \in T$.

To improve the Lagrangian decomposition each subproblem is tightened. For the productioninventory subproblem the following valid inequalities are considered.

$$
\begin{array}{ll}
\sum_{\ell=1}^{t} y_{\ell} \geq\left\lceil\frac{1}{\bar{P}_{t}}\left(\sum_{i \in N_{c}} \sum_{\ell=1}^{t} d_{i \ell}-\sum_{i \in N} S_{i}^{0}\right)\right] & \forall t \in T, t>1 \\
\sum_{\ell=1}^{t} z_{i \ell}^{1} \geq\left\lceil\frac{1}{\min \left\{\bar{I}_{i}, Q\right\}} \sum_{\ell=1}^{t}\left(d_{i \ell}-S_{i}^{0}\right)\right] & \forall i \in N_{c}, \forall t \in T
\end{array}
$$

Inequalities (22) impose a minimum number of production periods, while inequalities (23) impose a minimum number of visits to each client $i$.

For the routing subproblems we consider the Miller-Tucker-Zemlin [18] reformulation. We introduce new variables $w_{i t}$ indicating the load on the vehicle when client $i \in N_{c}$ is visited in period $t \in T$ (before delivering), and include the classical set of constraints,

$$
\begin{array}{ll}
w_{i t} \geq w_{j t}+q_{i t}-Q\left(1-x_{i j t}\right), & \forall(i, j) \in A, \forall t \in T, \\
w_{i t} \leq Q z_{i t}, & \forall i \in N_{c}, \forall t \in T, \\
w_{i t} \geq 0, & \forall i \in N_{c}, \forall t \in T .
\end{array}
$$


Inequalities (24)-(26) are added to each routing subproblem.

\section{Computational Results}

A computational experimentation was conducted to test the bounds provided by the two Lagrangian approaches. The computational tests were conducted using the Xpress-Optimizer 28.01.04 solver with the default options.

First we provide details on the generation of benchmark instances. The coordinates of the clients are randomly generated in a 100 by 100 square grid, and the producer is located in the center of the grid. For the number of clients two values are considered: $n=10$, and $n=20$. For the number of periods three values are considered: $n t=5, n t=10$, and $n t=15$. For each pair of values $n, n t$, three instances are generated, giving a total of 18 instances.

For each value of $n$, a complete graph with a symmetric traveling cost matrix associated to the set of arcs is considered. The traveling costs $C_{i j}$ and $C_{j i}$ are the Euclidean distance between the nodes $i$ and $j$ in the grid. The demand values, $d_{i t}$, are randomly generate between 40 and 80 units. The initial stock at producer $S_{0}^{0}$ is zero, and the initial stock $S_{i}^{0}$ at client $i$ is randomly generated between 0 and three times the average demand of client $i$. The holding cost $H_{i}$ is one for all $i \in N$. The maximum inventory level $\bar{I}_{i}$ is 500 for all $i \in N$. The production capacity $\bar{P}_{t}$ is $50 \%$ of the average demand. The production set up cost and the unit production cost are given by $S C=100$ and $P C=1$, respectively. The number of available vehicles is $m=3$. The vehicle usage cost is $V C=50$, and the vehicle capacity is $Q=500$.

Table 1 reports the instance data and the bounds obtained through the computational experiments. First column displays the number $n$ of clients, the second column displays the number $n t$ of periods, and the third column is the instance identifier number for each pair $n, n t$. The next columns report the results obtained with the linear programming relaxation of model PIR (columns $L P$ ), the Lagrangian relaxation given in Section 3 (columns $L R$ ), the Lagrangian decomposition given in Section 4 with all enhancements (columns ILD) and the best upper bound obtained using the branch and bound procedure from the solver Xpress to solve the PIR model given in Section 2 with a time limit of 2 hours (column $U B$ ). None of the tested instances could be solved to optimality within the given running time limit. For columns $L P$ we report the value of the lower bound $(L B)$ and the running time (Time). For columns $L R$ and $I L D$ we report the value of the lower bound $(L B)$, the number of iterations of the subgradient algorithm (Iter), and the running time (Time).

We can see that the linear relaxation is fast. The lower bounds obtained using the Lagrangian relaxation are lower than the lower bounds obtained using the linear relaxation. In theory one should observe the opposite relation between bounds when the optimal multipliers are used. This means that we could not identify near optimal multipliers. The Lagrangian decomposition provides always the best lower bounds. The Lagrangian relaxation terminates when the maximum number of iterations (500) was attained, while the Lagrangian decomposition terminates, in most of the cases, when the time limit of 20 minutes was attained. Thus, one may conclude that solving the Lagrangian decomposition at each iteration of the subgradient algorithm requires high running times (as expected, since more complex subproblems are solved in each iteration) but provides better lower bounds. 
Table 1: Comparison of bounds obtained via Lagrangian approaches.

\begin{tabular}{ccc|cc|ccc|ccc|c}
\multicolumn{1}{|c|}{ LP } & \multicolumn{9}{|c}{ LR } \\
\hline \multicolumn{1}{l|}{$n$} & nt & Inst. & LB & Time & LB & Iter & Time & LB & Iter & Time & \\
\hline 10 & 5 & 1 & 1736.5 & 0 & 1496.0 & 500 & 19.5 & 2338.8 & 41 & 56.3 & 2754 \\
10 & 5 & 2 & 1491.6 & 0 & 1236.4 & 500 & 19.4 & 1690.2 & 16 & 37.9 & 2637 \\
10 & 5 & 3 & 1491.9 & 0 & 1240.5 & 500 & 19.2 & 1910.8 & 25 & 34.7 & 2835 \\
10 & 10 & 1 & 2864.2 & 0 & 2531.9 & 500 & 72.2 & 4604.7 & 33 & 167.8 & 5603 \\
10 & 10 & 2 & 2730.3 & 0 & 2247.6 & 500 & 74.0 & 3618.9 & 13 & 89.0 & 5464 \\
10 & 10 & 3 & 2673.0 & 0 & 2215.5 & 500 & 71.9 & 3678.9 & 20 & 111.0 & 5692 \\
10 & 15 & 1 & 3452.2 & 0.1 & 3070.6 & 500 & 251.3 & 6640.9 & 27 & 202.8 & 8261 \\
10 & 15 & 2 & 3603.6 & 0.1 & 2855.9 & 500 & 250.5 & 5294.8 & 17 & 169.5 & 7828 \\
10 & 15 & 3 & 3928.7 & 0.1 & 3262.2 & 500 & 251.2 & 5588.3 & 18 & 151.3 & 8729 \\
20 & 5 & 1 & 2504.5 & 0.1 & 1630.8 & 500 & 122.5 & 2805.6 & 14 & 451.6 & 3919 \\
20 & 5 & 2 & 2670.6 & 0.1 & 1952.7 & 500 & 124.4 & 2618.4 & 15 & 550.5 & 4252 \\
20 & 5 & 3 & 2917.4 & 0.1 & 2123.5 & 500 & 118.0 & 2970.2 & 17 & 336.6 & 4438 \\
20 & 10 & 1 & 4535.9 & 0.3 & 2809.9 & 500 & 239.4 & 5665.7 & 17 & 1213.3 & 7914 \\
20 & 10 & 2 & 4116.7 & 0.3 & 2514.2 & 500 & 238.7 & 4791.9 & 15 & 1283.2 & 7644 \\
20 & 10 & 3 & 4571.8 & 0.3 & 2788.3 & 500 & 241.7 & 5676.0 & 22 & 683.2 & 8805 \\
20 & 15 & 1 & 6230.2 & 0.4 & 3749.2 & 500 & 493.8 & 7578.9 & 8 & 1253.5 & 12067 \\
20 & 15 & 2 & 5917.3 & 0.5 & 3403.5 & 500 & 498.3 & 6890.4 & 10 & 1235.4 & 11785 \\
20 & 15 & 3 & 6739.8 & 0.4 & 4091.3 & 500 & 494.9 & 8464.0 & 18 & 1066.3 & 13335
\end{tabular}

\section{Conclusions}

We consider a complex production-inventory-routing problem. For this problem two Lagrangian approaches are tested. One is a classical Lagrangian relaxation that allows to split the problem into several small subproblems, where two of them can be solved by inspection. The other approach decomposes the problem into two main subproblems (one is a productioninventory problem and the other is a routing problem). Such subproblems are still complex and NP-hard. Nevertheless, they can be solved for reasonable size instances using a commercial solver. The computational tests indicate that keeping a higher degree of complexity in the subproblems leads to harder subproblems, requiring larger running times to solve to optimality, but allow us to derive better lower bounds. Also, as the quality of the lower bounds obtained through Lagrangian approaches depend greatly on the quality of the Lagrangian multipliers, the computational results show that finding near optimal multipliers is a challenging task when we consider the classical approach that uses many multipliers. Overall we may conclude that, when the subproblems can be solved to optimality, then it may be preferable to consider such complex subproblems and perform less iterations of a subgradient method using a few number of Lagrangian multipliers, than to consider simpler subproblems which allow to perform more iterations, but require the tuning of an high number of Lagrangian multipliers. As future research we aim to investigate decomposition approaches that are based on similar decomposition ideas as the one we tested here, but where no computation of Lagrangian multipliers is required.

\section{Acknowledgements}

The research of the first and third authors was supported through CIDMA and FCT, the Portuguese Foundation for Science and Technology, within project UID/MAT/ 04106/2013. 
The research of the second author was financed by the ERDF - European Regional Development Fund through the Operational Programme for Competitiveness and Internationalisation - COMPETE 2020 Programme within project "POCI-01-0145-FEDER-006961", and by FCT within project UID/EEA/50014/2013.

\section{References}

1. Adulyasak, Y., Cordeau, J., Jans, R.: The production routing problem: A review of formulations and solution algorithms. Computers \& Operations Research 55, 141-152 (2015)

2. Adulyasak Y., Cordeau J., Jans, R.: Formulations and branch and cut algorithms for multi-vehicle production and inventory routing problems. INF J Comput, 26(1), 103-120 (2014)

3. Agra, A., Andersson, H., Christiansen, M., Wolsey, L.: A maritime inventory routing problem: Discrete time formulations and valid inequalities. Networks 62, 297-314 (2013)

4. Agra, A., Christiansen, M., Delgado, A.: Mixed Integer Formulations for a Short Sea Fuel Oil Distribution Problem. Transportation Science 47, 108-124 (2013)

5. Agra, A., Christiansen, M., Delgado, A., Simonetti, L.: Hybrid heuristics for a short sea inventory routing problem. European Journal of Operational Research 236, 924-935 (2014)

6. Agra, A., Christiansen, M., Ivarsoy, K., Solhaug, I., Tomasgard, A.: Combined ship routing and inventory management in the salmon farming industry. Annals of Operations Research (in press)

7. Andersson, H., Hoff, A., Christiansen, M., Hasle, G., Løkketangen, A.: Industrial aspects and literature survey: Combined inventory management and routing. Computers \& Operations Research, 37(9), 1515-1536 (2010)

8. Archetti, C., Bertazzi, L., Laporte, G., Speranza, M.G.: A branch-and-cut algorithm for a vendormanaged inventory-routing problem. Transportation Sci. 41(3), 382-391 (2007)

9. Bell, W.J., Dalberto, L.M., Fisher, M.L., Greenfield, A.J., Jaikumar, R., Kedia, P.: Improving the distribution of industrial gases with an on-line computerized routing and scheduling optimizer Interfaces, 13(6), 4-23 (1983)

10. Christiansen, M.: Decomposition of a combined inventory and time constrained ship routing problem. Transportation Science, 33(1), 3-16 (1999)

11. Christiansen, M., Fagerholt, K.: Maritime Inventory Routing Problems. In Floudas, C., Pardalos, P. (Eds.), Encyclopedia of optimization, second edition. Springer, 1947-1955 (2009)

12. DuniEksioglu, S., Pardalos, P., Romeijn, H.E.: Cross-Facility Management of production and Trasportation Planning Problem. Computers and Operations Research, 33(11), 3231-3251 (2006)

13. Eppen, G.D., Martin, R.K.: Solving multi-item capacitated lot-sizing problems using variable redefinition. Operations Research 35, 832-848 (1997)

14. FICO Xpress Optimization Suite

15. Fumero, F., Vercellis, C.: Synchronized development of production, inventory, and distribution schedules. Transp Sci, 33 (3), 330-340 (1999)

16. Geunes, J., Pardalos, P.M.: Network Optimization in Supply Chain Management and Financial Engineering: An Annotated Bibliography. Networks, 42 (2), 66-84 (2003)

17. Held, M., Wolfe, P., Crowder, H.P.: Validation of subgradient optimization. Mathematical Programming, 6, 62-88 (1974)

18. Miller, C., Tucker, A., Zemlin, R.: Integer programming formulations and travelling salesman problems. Journal of the Association for Computing Machinery, 7(4), 326-329 (1960)

19. Pei, J., Pardalos, P.M., Liu, X., Fan, W., Yang, S., Wang, L.: Coordination of production and transportation in supply chain scheduling. Journal of Industrial and Management Optimization, 11 (2), 399-419 (2015)

20. Ruokokoski, M., Solyali, O., Cordeau, J.-F., Jans, R., Süral, H.: Efficient formulations and a branch-and-cut algorithm for a production-routing problem. GERAD Technical Report G-201066, HEC Montréal, Canada (2010)

21. Shor, N.Z.: Minimization Methods for Non-Differentiable Functions. Springer-Verlag, English translation (1985) 
22. Solyali, O., Süral, H.: A branch-and-cut algorithm using a strong formulation and an a priori tour-based heuristic for an inventory-routing problem. Transportation Sci. 45(3), 335-345 (2011) 23. Solyali, O., Süral, H.: A relaxation based solution approach for the inventory control and vehicle routing problem in vendor managed systems. S.K. Neogy, A.K. Das, R.B. Bapat (Eds.), Modeling, computation and Optimization, World Scientific, Singapore, Sinagpore, 171-189 (2009)

24. Solyali, O., Süral, H.: The one-warehouse multi-retailer problem: Reformulation, classification and computational results. Annals of Operations Research 196(1), 517-541 (2012) 